\title{
17.
}

\section{Über das Verhalten der Gamma-Functionen zu den Producten äquidifferenter Factoren.}

\author{
(Von Herrn Prof. Dr. M. Ohm zu Berlin.)
}

\section{\$. 1.}

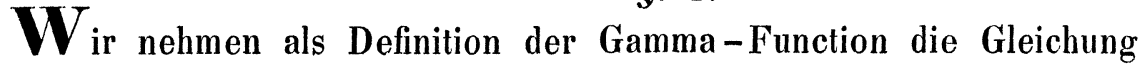

$$
\text { I. } T_{x}=\int_{0}^{\infty} e^{-z} \cdot z^{x-1} \cdot d z=\int_{0}^{1}\left(\log \frac{1}{z}\right)^{x-1} \cdot d z,
$$

in welcher wir $x$ stets positiv (ganz oder gebrochen) voraussetzen.

Ist $x$ positiv ganz, so findet sich durch theilweise Integration unmittelbar

II. $T_{x}=1 \cdot 2 \cdot 3 \ldots(x-1)$, so wie $I_{2}=1$ und $I_{1}=1$.

Ist aber $x$ gebrochen, so verhält sich die transcendente Gamma-Function $I_{x}$ zu dem Product äquidifferenter Factoren, wie sich die (transcendente) Exponential-Function $e^{x}$ zu dem Producte gleicher Factoren verhält; und wie wir von der Betrachtung eines Productes gleicher Factoren ausgehen, um nach und nach zu den (transcendenten) Exponenlial-Functionen und deren Eigenschaften zu gelangen, so ist es naturgemäfs, von den Producten äquidifferenter Factoren auszugehen, um in ihren Gesetzen, auch die Grundgesetze und die wesentlichsten Eigenschaften der Gamma-Functionen bereits ausgesprochen zu erblicken.

$$
\text { \$. } 2 .
$$

Wir definiren nun

III. $\quad a^{n \mid r}=a(a+r)(a+2 r) \ldots(a+(n-1) \cdot r)$,

wo $n$ positiv ganz gedacht ist, nennen $a^{n \mid r}$ eine ganze Factorielle, so wie $a$ die Basis, $n$ den Exponenten und $\boldsymbol{r}$ die Differenz derselben, und wir ziehen aus dieser Definition die Folgerungen

$$
\begin{array}{rlrl}
\text { IV. } & \boldsymbol{a}^{n \mid r} & =(\boldsymbol{a}+(\boldsymbol{n}-\mathbf{1}) \cdot \boldsymbol{r})^{n \mid-r} ; \\
\text { V. } & \boldsymbol{a}^{m+n \mid r} & =\boldsymbol{a}^{m \mid r} \cdot(\boldsymbol{a}+\boldsymbol{m} \boldsymbol{r})^{n \mid r}=\boldsymbol{a}^{n \mid r} \cdot(\boldsymbol{a}+\boldsymbol{n} \boldsymbol{r})^{m \mid r}, \\
\text { VI. } & \boldsymbol{a}^{m-n \mid r} & =\frac{\boldsymbol{a}^{m \mid r}}{(\boldsymbol{a}+(\boldsymbol{m}-\boldsymbol{n}) \cdot \boldsymbol{r})^{n \mid r},} \\
\text { VII. } & & \frac{\boldsymbol{a}^{m \mid r}}{\boldsymbol{a}^{n \mid r}} & =(\boldsymbol{a}+\boldsymbol{n} \boldsymbol{r})^{m-n \mid r} ;
\end{array}
$$

Crelle's Journal f. d. M. Bd. XXXVI. Heft 4. 
so wie (aus (V.), wenn $a+n r=b$ gesetzt wird)

$$
\text { VIII. } \quad \frac{a^{m \mid r}}{b^{m \mid r}}=\frac{a^{(b-a): r \mid r}}{(a+m \cdot r)^{(b-a): r \mid r}},
$$

(wo aber alle Exponenten als positiv ganz vorausgeselzt werden, namentlich also auch $\left.\frac{b-a}{r}\right)$;

also auch

$$
\text { IX. } \quad a^{m \mid r}=\left(\frac{a}{h}\right)^{m \mid \frac{r}{h}} \cdot h^{m},
$$

$$
\text { IX. b. } \quad a^{m \mid r}=r^{m} \cdot\left(\frac{a}{r}\right)^{m \mid 1}=r^{m} \cdot \frac{1^{(a: r)+m-1 \mid 1}}{1^{(a: r)-1 \mid 1}},
$$

durch welche letztere Gleichung die Factorielle $a^{m \mid r}$ auf den einfachsten Fall derselben, wo die Basis und die Differenz gleichmäfsig der Einheit gleich sind, zurückgeführt sich sieht.

In allen diesen Gleichungen setzen wir aber die Exponenten positiv ganz voraus, damit wir es überall nur mit Producten äquidifferenter Factoren zu thun haben.

$$
\text { S. } 3 .
$$

Nun aber nimmt man die Gleichung (VI.) als Definition der Differen:Factorielle $a^{m-n \mid r}$, deren Exponent $m-n$ eben so gut positiv als auch negativ ganz gedacht wird, eben so gut Null als 1. - Man untersucht aber mit Sorgfalt und findet zu Folge dieser Untersuchungen, dafs für diesen erweiterten Begriff der Factorielle $a^{b \mid r}$ die Formeln (IV. - IX.) noch gelten, obgleich jetzt die Factoriellen Quotienten aus Producten äquidifferenter Factoren vorstellen. Die Exponenten dieser Factoriellen sind also jetzt positive oder negative ganze Zahlen, oder Null; die Basis und die Differenz einer jeden ist aber beliebig, reell oder imaginär.

In der Formel (VI.) steckt aber nun noch

$$
\begin{array}{rlrl}
\text { X. } & & a^{1 \mid r}=a ; \\
\text { XI. } & a^{\mid \eta r}=1 ; \\
\text { XII. } & a^{-n \mid r}=\frac{1}{(a-n r)^{n \mid r}}=\frac{1}{(a-r)^{n \mid-r}},
\end{array}
$$

während $n$ selbst beliebig positiv oder negativ ganz oder Null ist. Endlich ist auch noch für diese allgemeinere Factorielle:

XIII. $\quad a^{m \mid 0}=a^{m}$,

so dafs die Gesetze dieser Factoriellen in die Gesetze der Potenzen (mit po- 
sitiven oder negativen ganzen oder Null-Exponenten) übergehen, so oft man die Differenz $r,=0$ setzt.

\$. 4.

Hierauf nehme man aus der (II.) und der (IX.b.) diese drei Definitionen:

XIV. $\quad 1^{c \mid 1}=\Gamma_{1+c}$ d. h. $=\int_{0}^{\infty} e^{-z} \cdot z^{c} \cdot d z=\int_{0}^{1}\left(\log \frac{1}{z}\right)^{c} \cdot d z$, wo $1+c$ positiv gedacht ist;

$\mathrm{XV} . \quad \boldsymbol{a}^{c \mid 1}=\frac{\boldsymbol{\Gamma}_{a+c}}{\boldsymbol{\Gamma}_{a}}$,

wo $a$ und $a+c$ positiv gedacht sind, und welche die (XIV.) in sich schliefst, XVI. $\quad a^{c \mid r}=r^{c} \cdot \frac{\Gamma_{(a: r)+e}}{\Gamma_{(a: r)}}$,

wo $r$ und $a$ und $\frac{a}{r}+c$ positiv gedacht sind, während $c$ beliebig ganz oder gebrochen sein soll. - Im letztern Falle nennt man die Factoriellen gebrochen.

So wie aber diese Begriffe festgestellt sind, mufs sogleich wieder eine Untersuchung angestellt werden, und diese lehrt, dafs die vorhergehenden Formeln (V. - XII.) für gebrochene Factoriellen ebenfalls noch gelten, welche reelle Werthe auch die Buchstaben nur immer vorstellen, wenn nur die Bedingungen der Existenz dieser gebrochenen Factoriellen erfüllt sind, - da/s aber die (IX.), nämlich

$$
\boldsymbol{a}^{m \mid r}=\left(\frac{a}{h}\right)^{m \mid \frac{r}{h}} \cdot h^{m}
$$

nur dann gilt, wenn $h$ positiv ist und $h^{m}$ ihren positiven Werth vorstellt; während von der (IV.) deshalb zur Zeit in Bezug auf gebrochene Factoriellen keine Rede sein kann, weil bis jetzt bei den gebrochenen Factoriellen nur positive Differenzen vorausgesetzt worden sind.

S. 5.

Um nun aber auch Factoriellen mit beliebig grofsem negativem Exponenten zu haben, nehme man aus der (V.) als Definition

XVII. $\quad a^{c \mid r}=\frac{a^{\prime \mid r}}{(a+c r)^{v \mid r}} \cdot(a+\nu r)^{c \mid r}=\frac{a^{\nu \mid r}}{(a+c r)^{|| r}} \cdot r^{c} \cdot \frac{\Gamma_{(a: r)+c+\nu}}{\Gamma_{(a: r)+\nu}}$,

nachdem man sich $\nu$ positiv ganz und grofs genug gedacht hat, dafs, wenn auch $a$ und $c$ beliebig grofs und negativ sein sollten, doch $\frac{a}{r}+\nu$ und $\frac{a}{r}+c+v$ stets positiv werden, während die Differenz $r$ immer nur positiv vorausgesetzt wird. 
Dann aber untersucht und findet man aufs Neue, dafs alle vorhergehenden Formeln für diese jetzt ziemlich allgemeinen Factoriellen auch noch gelten, jedoch die (IX.) wiederum nur, wenn h positiv ist und die Potenz $h^{\text {m }}$ ilhren positiven Werth vorstellt. - Von der (IV.) endlich kann jedoch für gebrochene Exponenten wiederum zur Zeit deshalb noch nicht die Rede sein, weil wir bis jelzt noch keine weitern Factoriellen kennen, als solche, die nur positive Differen hen haben. - Die Exponenten und Basen können dagegen beliebiy reell gedacht werden.

\section{\$. 6 .}

Endlich nehmen wir die Gleichung (IV.) als Definition der gebrochenen (oder ganzen) Factorielle mit negativer Differenz (indem wir in (IV.) $a-(n-1) r$ stalt $\iota$ schreihen). - Nachdem diese letztere Definition noch hinzugekommen, hat aber die Factorielle $a^{c \mid r}$ jedesmal eine völlig bestimmte Bedeutung, sie hat jedesmal einen bestimmten einzigen und reellen Werth, wie auch $a$ und $r$ und $c$ beliebig reell gegeben sein mögen *).

Untersucht man aber, so findet man auch in Bezug auf die letztere Definition, dafs alle vorhergehenden Formeln, die nicht ihrer Natur nach ganz specielle sind, für alle Factoriellen gelten, während alle vorkommenden Buchstaben beliebige reelle Werthe haben, mit Ausnahme der (IX.), die nur yilt, wenn $h$ positiv ist und $h^{m}$ positiv genommen wird $\left.{ }^{* *}\right)$.

\section{S. 7.}

Ist $\nu$ positiv ganz, $c$ aber wie $a$ beliebig reell und endlich, so nähert sich der Quotient $\frac{\nu^{c}}{(a+\nu)^{c \mid}}$ der 1 desto mehr, je gröfser $\nu$ gedacht wird, und man hat

$$
\text { XVIII. } \frac{\nu^{c}}{(a+v)^{c \mid 1}}=1 \text { für } \quad \nu=+\infty .
$$

Für ganze Werthe von $c$ fällt die Wahrhe;t dieser Behauptung in die Augen; und für gebrochene Werthe von $c$ ist, wegen

$$
\text { 1. }(a+v)^{c \mid 1}=\frac{\Gamma_{a+v+c}}{\Gamma_{a+\nu}},
$$

*) Dafs sich immer in den besondern Fällen der Anwendung die Fälle als Ausnahmsfälle ausscheiden, in welchen Ausdrücke vorkommen, welche die Form $\frac{1}{6}$ annehmen, versteht sich von selbst.

**) Dafs Kramp diese Formel (IX.) für allgemein wahr hält und sie auch anwendet, wenn $h$ negativ ist, kann man als die Hauptquelle der Widersprüche ansehen, in welche er sich in seiner „Analyse des réfraclions astron. et terrestres 1799" verwickelt sieht. 
noch

$$
\text { 2. } \frac{v^{c}}{(a+\nu)^{c \mid 1}}=\frac{\nu^{c} \cdot \Gamma_{a+\nu}}{\Gamma_{a+\nu+c}}=\frac{\nu_{0}^{c} \cdot \int_{0}^{\infty} e^{-z} \cdot z^{a+\nu-1} \cdot d z}{\int_{0}^{\infty} e^{-z} \cdot z^{a+\nu+c-1} \cdot d z} \text {. }
$$

Nun hat man aber für $\nu=+\infty, e^{-z}=\left(1-\frac{z}{\nu}\right)^{\nu}$, weil beide Seiten einerlei Logarithmen geben, wenigstens für jeden endlichen Werth von $z$, und weil für $\boldsymbol{z}=\infty \overline{\bar{\chi}} \boldsymbol{\nu}$ beide Seiten unendlich klein werden. Und da ferner der aggregirende Theil der beiden Integrale zur Rechten, der noch hinzutreten mufs, wenn solche zuerst von $z=0$ bis $z=\nu$, dann aber noch von $z=v$ bis zu jedem noch gröfsern Werth von $z$ genommen werden, für $v=\propto$ offenbar der Null gleich sind, so kann man statt der obern Grenze $\propto$ der Integrale jedesmal $\nu$ schreiben (wenn $\nu=+\infty$ ), so dafs die Gleichung (2.) dadurch übergeht in

$$
\text { 3. } \frac{v^{c}}{(a+\nu)^{c \mid 1}}=\frac{v_{0}^{c} \cdot \int_{0}^{v}\left(1-\frac{z}{\nu}\right)^{v} \cdot z^{a+v-1} \cdot d r}{\int_{0}^{v}\left(1-\frac{z}{v}\right)^{v} \cdot z^{a+v+c-1} \cdot d z}
$$

oder, wenn man $\frac{z}{\nu}=x$ setzt:

$$
\text { 4. } \frac{\nu^{c}}{(a+\nu)^{c \mid 1}}=\frac{\int_{0}^{1}(1-x)^{\nu} \cdot x^{a+\nu-1} \cdot d x}{\int_{0}^{1}(1-x)^{\nu} \cdot x^{a+\nu+c-1} \cdot d x},
$$

für $v=+\infty$. - Dafs aber das Verhältnifs (der Quotient) dieser letztern beiden Integrale desto näher der Einheit rückt, je gröfser $\nu$ gedacht wird, und für $v=+\infty$ der Einheit unendlich nahe kommt, ist unschwer zu erkennen.

Anmerkung. Aus diesem Satze folgt noch

$$
\text { XIX. } \quad a^{c \mid+\frac{1}{\infty}}=a^{c} \text {, }
$$

so lange a positiv ist. - Denn es ist

$$
a^{c \mid r}=r^{c} \cdot\left(\frac{a}{r}\right)^{c \mid 1}
$$

folglich auch

$$
\frac{a^{c}}{a^{c \mid r}}=\left(\frac{a}{r}\right)^{c}:\left(\frac{a}{r}\right)^{c \mid 1}=\frac{v^{c}}{v^{c \mid 1}}
$$

wenn $\frac{a}{r}=\nu$ gesetzt wird. Weil nun aber $\nu$ positiv unendlich grofs wird, so oft $r=+\frac{1}{\infty}$ und $a$ positiv gedacht wird, so folgt das Behauptete. 
Man findet aber auch noch

XIX. $b$.

$$
a^{c \mid-\frac{1}{\infty}}=a^{c}
$$

wenn nur a positiv und endlich ist.

Denn es ist $a^{c \mid-\frac{1}{\infty}}=\left(a-(c-1) \cdot \frac{1}{\infty}\right)^{c \mid+\frac{1}{\infty}}=\left(a-(c-1) \cdot \frac{1}{\infty}\right)^{c}=a^{c}$.

Deshalb kann man annehmen, dafs die (XIII.), nämlich $a^{m \mid 0}=a^{m}$, auch noch für jeden gebrochenen reellen Werth von $m$ gilt, so lange nur a positiv ist.

\$. 8.

Die Gleichung (V.) giebt daher für $r=1$ :

$$
\text { XX. } \quad a^{c \mid 1}=\frac{a^{\nu \mid 1}}{(a+c)^{\nu \mid 1}} \cdot \nu^{c} \quad \text { für } \quad \nu=+\infty,
$$

wo $a$ und $c$ beliebig reell gedacht sind. - Für $a=1$ geht diese Gleichung über in

$$
\text { XXI. } \quad 1^{c \mid 1}=\frac{1^{v \mid 1}}{(1+c)^{\nu \mid 1}} \cdot \nu^{c} \quad \text { für } \quad \nu=+\infty,
$$

wo $c$ beliebig reell gedacht ist ${ }^{*}$ ).

Multipliciren wir in (XX.) rechts Zähler und Nenner mit $\boldsymbol{r}^{\nu}$, und die Gleichung selbst mit $\boldsymbol{r}^{c}$, und setzen wir $\boldsymbol{r}$ positiv voraus, so ergiebt sich, wenn noch $\frac{a}{r}$ statt $a$ gesetzt wird (nach IX.):

$$
\text { XXII. } \quad a^{c \mid r}=\frac{a^{\nu \mid r}}{(a+c r)^{\nu \mid r}} \cdot(\nu r)^{c} \quad \text { für } \quad v=+\infty,
$$

dagegen ist, wenn wiederum $r$ positiv, also $-r$ negativ gedacht wird,

$$
\text { XXIII. } \quad a^{c \mid-r}=\frac{a^{-v \mid r}}{(a+c r)^{-\nu \mid r}} \cdot(\nu r)^{c} \quad \text { für } \quad \nu=+\infty,
$$

welche Formel mittelst der (IV.) aus der (XXII.) unmittelbar hervorgeht.

Anmerkung. Man begreift übrigens, dafs man von diesen letztern beiden Gleichungen als Definition der gebrochenen Factoriellen mit positiver, dann auch mit negativer Differenz, hätte ausgehen können. Dann würde sich aus diesen Definitionen die Gültigkeit der Formeln (IV. - XVII.) haben ab-

*) Diesen Ausdruck zur Rechten in (XXI.) hat Gaufs in der Abhandlung vom Jahre 1812: „Disquis. gener. circa seriem infinitam etc." im 2ten Bande der Göttinger Commentarien durch $\Pi_{c}$ bezeichnet. Es ist also $\Pi_{c}$ völlig identisch mit $1^{c \mid 1}$, aber allgemeiner als $\Gamma_{1+c}$, so lange wir unter $\Gamma_{c}$ nichts anderes als das bestimmte Integral in (I.) verstehen, weil $\Pi_{c}$ nur dann $=\Gamma_{1+c}$ ist, wenn $c$ zwischen -1 und $\infty$ liegt, während $\mathbb{1}^{c \mid 1}$ oder $\boldsymbol{I}_{c}$ für jeden andern negativen Werth von $c$ auch seine Bedeutung hat. 
leiten lassen; aber auch da würde man gefunden haben, dafs die (IX.) nur gilt, wenn $h$ und $h^{m}$ positiv sind. - Wir werden diesen bessern Weg bei einer andern Gelegenheit (in einer eignen Schrift) nachweisen. -

S. 9.

Das Verhältnifs (der Quotient) $\frac{a^{c \mid r}}{b^{c \mid r}}$ zweier Factoriellen, welche denselben Exponenten und dieselbe Differenz haben, läfst sich auf 8 verschiedene Arten unmittelbar so umformen (wenn man die (VIII.) und dann auf jede der 4 Factoriellen die (XIII.) und die (IV.) anwendet), dafs immer Zähler und Nenner dieselbe Differenz und denselben Exponenten haben, aber mit allen Combinationen der Vorzeichen. Man hat nämlich :

$$
\begin{gathered}
\frac{a^{c \mid r}}{b^{c \mid r}}=\frac{(b-r)^{-c \mid-r}}{(a-r)^{-c \mid-r}}=\frac{(b+c r)^{-c \mid r}}{(a+c r)^{-c \mid r}}=\frac{(a+(c-1) \cdot r)^{c \mid-r}}{(b+(c-1) \cdot r)^{c \mid-r}} \\
=\frac{a^{(b-a): r \mid r}}{(a+c r)^{(b-a): r \mid r}}=\frac{(a+(c-1) \cdot r)^{(a-b): r \mid-r}}{(a-r)^{(a-b): r \mid-r}}=\frac{(b+c r)^{(a-b): r \mid r}}{b^{(a-b): r \mid r}}=\frac{(b-r)^{(b-a): r \mid-r}}{(b+(c-1) \cdot r)^{(b-a): r \mid-r}} .
\end{gathered}
$$

Wenn man sich diese 8 Umformungen wohl einprägt, so wird man bei dem Rechnen mit Factoriellen kaum mehr Schwierigkeiten begegnen.

S. 10.

Der Haupt-Eigenschaft der Factoriellen, welche in der Gleichung (V.), nämlich in der Gleichung

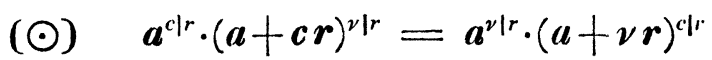

oder (für $r=1$ und $a=1$, desgleichen $c-1$ statt $c$ )

$$
c^{v \mid 1}=\frac{1^{v \mid 1}}{1^{c-1 \mid 1}} \cdot(1+v)^{c-1}
$$

ausgesprochen ist, kann man sich nun, wenn c gebrochen, $v$ aber ganz gedacht wird (beide aber positiv oder negativ) bedienen, einmal

um gebrochene Factoriellen in ganze auszudrücken, wie solches (\$. 8 . XX., XXII. und XXIII.) geschehen,dann aber auch

um ganze Factoriellen in gebrochene auszudrücken, also auch in GummaFunctionen (nach \$. 5. XV. und XVI.).

Dies letztere ist nun die Quelle der wichtigsten Eiyenschaften der Gamma-Functionen.

Jede einfachste Wahrheit nämlich, die durch eine Gleichung zwischen Producten äquidifferenter Factoren ausgedrückt ist, geht dadurch, dafs man die 
yanzen Factoriellen (nach der Formel $(\odot)$ oder $(\varangle)$ ) in gebrochene umformt, in eine Vergleichung der Gamma-Functionen über, so lange nur die Bedingungen der Existenz der Gamma-Functionen erfüllt sind. - Jede solche elementarste Wahrheit liefert also eine Eigenschaft der transcendenten GammaFunctionen.

Wir wollen dies jetzt an einigen Beispielen nachweisen.

\$. 11.

Erstes Beispiel. Es ist bekannt, dafs sich $\sin a \pi$ in ein Product aus unendlich vielen Factoren ausdrücken läfst. Nimmt man das analoge Product für $\sin b \pi$, dividirt man beide durch einander und hebt man so viel wie möglich auf, so erhält man:

$$
\text { 1. } \frac{\sin a \pi}{\sin b \pi}=\frac{a^{\nu \mid 1} \cdot(1-a)^{\nu \mid 1}}{b^{v \mid 1} \cdot(1-b)^{\nu \mid 1}} \text { für } \quad v=+\infty .
$$

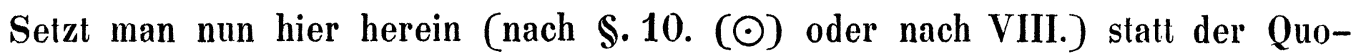
tienten $\frac{a^{\nu \mid 1}}{b^{\nu \mid 1}}$ und $\frac{(1-a)^{\left.\nu\right|^{1}}}{(1-b)^{\nu \mid 1}}$ die ihnen bezüglich gleichen Quotienten $\frac{a^{b-a \mid 1}}{(a+v)^{b}-\alpha \mid 1}$, und $\frac{(1-a)^{a-b \mid 1}}{(1-a+v)^{a-b \mid 1}}$ und bedenkt man, dafs weil $y=\infty$ die letztern beiden Nenner (nach \$. 7. XVIII.) bezüglich die Potenzen $\nu^{b-a}$ und $\nu^{a-b}$ sind, deren Product 1 ist, so erhält man aus (1.) sogleich

$$
\text { XXIV. } \frac{\sin a \pi}{\sin b \pi}=a^{b-a \mid 1} \cdot(1-a)^{a-b \mid 1},
$$

also auch, im Falle $b$ und $a$ positiv und kleiner als 1 vorausgesetzt werden, (nach \$. 4. XV.):

$$
\text { XXV. } \quad \frac{\sin a \pi}{\sin b \pi}=\frac{\Gamma_{b} \cdot \Gamma_{1-b}}{\Gamma_{a} \cdot \Gamma_{1-a}} .
$$

Setzt man hier $b=a+\frac{1}{2}$, so erhält man

$$
\text { XXVI. } \quad \operatorname{tang} a \pi=\frac{\Gamma_{\frac{2}{2}+a} \cdot \Gamma_{\frac{1}{2}-a}}{\Gamma_{a} \cdot \Gamma_{1-a}}
$$

wo aber a positiv und $<\frac{1}{2}$ vorausgesetzt werden mufs.

Und da (nach V. und XI.)

XXVII. $\quad a^{c \mid 1}=a \cdot(a+1)^{c-1 \mid 1}=a^{c-1 \mid 1} \cdot(a+c-1)$

ist, so folgt zunächst auch (für $a=1$ und $c$ positiv)

XXVIII. $\quad \Gamma_{c+1}=c \cdot \Gamma_{c}$;

und dann ergiebt sich noch, wenn man die (XXIV.) durch a dividirt und hier- 
auf $a=0$ setzt (in so fern $\frac{\sin a \pi}{a}=\pi$ wird, für $a=0$ )

$$
\text { XXIX. } \frac{\pi}{\sin 6 \pi}=1^{b-1 \mid 1} \cdot 1^{-b \mid 1},
$$

also auch (nach XIV.)

$$
\text { XXX. } \quad \frac{\pi}{\sin b \pi}=I_{b} \cdot I_{1-b},
$$

wenn nur $b<1$ und positiv ist. - Für $b=\frac{1}{2}$ wird

$$
\text { XXXI. } \quad \pi=\left(\boldsymbol{T}_{\frac{1}{2}}\right)^{2} \text { oder } \boldsymbol{I}_{\frac{1}{2}}=\sqrt{ } \pi \text {. }
$$

Und da (nach V.)

$$
1^{n+c-1 \mid 1}=1^{c-1 \mid 1} \cdot c^{n \mid 1}
$$

ist, so folgt hieraus noch (wegen XIV.)

$$
\text { XXXII. } \quad T_{n+e}=I_{c}^{\prime} \cdot c^{n \mid 1} \text {; }
$$

also auch (für $c=\frac{1}{2}$, wegen XXXI.)

$$
\text { XXXIII. } \quad I_{n+\frac{1}{2}}^{\prime}=\frac{1^{n / 2}}{2^{n}} \cdot \sqrt{ } \pi,
$$

welche Gleichungen hesonders dann von Interesse sind, wenn man sich $\boldsymbol{n}$ positiv ganz denkt, weil dann $c^{n \mid 1}$ und $1^{n \mid 2}$ Producte von $n$ Factoren vorstellen.

Setzt man ferner in der (XXX.) nach und nach statt $b$ erst $\frac{1}{n}$, dann $\frac{2}{n}, \frac{3}{n}$ etc. etc., zuletzt $\frac{n-1}{n},-$ multiplicirt man alle entsprechenden Gleichungen mit einander und bedenkt man, dafs

$$
\left.\sin \frac{1}{n} \pi \cdot \sin \frac{2}{n} \pi \cdot \sin \frac{3}{n} \pi \ldots \sin \frac{n-1}{n} \pi=\frac{n}{2^{n-1}}{ }^{*}\right)
$$

und

$$
I_{1}=1
$$

ist, so erhält man

$$
\text { XXXIV. } \quad I_{\frac{1}{n}} \cdot I_{\frac{2}{n}} \cdot I_{\frac{3}{n}}^{\prime} \ldots I_{\frac{n-1}{n}}^{\prime}=\frac{(2 \pi)^{\frac{1}{2}(n-1)}}{\sqrt{n}}
$$

U. s. w. f.

*) Zerlegt man die durch $\frac{x^{n}-1}{x-1}$ ausgedrückte ganze Function vom $n-1$ ten Grade in ihre $n-1$ einfachen Factoren (so dafs jeder Factor durch $x-\cos \frac{2 \mu}{n} \pi-i \cdot \sin \frac{2 \mu}{11} \pi$ vorgestellt ist, unter $i$ die $\sqrt{-1}$ und unter $\mu$ die ganzen Zahlen $1,2,3, \ldots n-1$ verstanden) und setzt man in der vorstehenden Gleichung 1 statt $x$, so erhält man

$$
n=\prod_{\mu=1}^{\mu=n-1}\left(1-\cos \frac{2 \mu}{n} \pi-i \cdot \sin \frac{2 \mu}{n} \pi\right) \text {. }
$$

Weil aber $1-\cos \frac{2 \mu}{n} \pi=2\left(\sin \frac{\mu}{n} \pi\right)^{2}$ und $\sin \frac{2 \mu}{n} \pi=2 \sin \frac{\mu}{n} \pi \cdot \cos \frac{\mu}{n} \pi$ ist, so geht

Crelle's Journal f. d. M. Bd. XXXVI. Heft 4. 
\$. 12.

Zweites Beispiel. Man gehe nun von der sehr elementaren Wahrheit aus, dafs das Product von $2 \nu$ Factoren

$$
a(a+1)(a+2)(a+3)(a+4) \ldots(a+2 v-1)
$$

sich zerlegt in das Product der $v$ Factoren $a(a+2)(a+4) \ldots(a+2 v-2)$, multiplicirt mit dem Producte der $v$ übrigen Factoren, d. h. also dafs ist

$$
\text { 1. } a^{2 \cdot 11}=a^{v / 2} \cdot(a+1)^{v / 2}
$$

oder (nach IX.)

$$
=\left(\frac{a}{2}\right)^{\nu \mid 1} \cdot 2^{\nu} \times\left(\frac{a+1}{2}\right)^{\nu \mid 1} \cdot 2^{\nu}
$$

d. h.

$$
\text { 2. } \quad \frac{a^{2 \nu \mid 1}}{\left(\frac{a}{2}\right)^{v \mid 1} \cdot\left(\frac{a+1}{2}\right)^{\nu / 1}}=2^{2 v} \text {, }
$$

dafs also der Quotient zur Linken (in N. 2.), von dem Werthe von $a$ ganz unabhängig ist.

Setzt man nun hier herein statt der ganzen Factoriellen, die ihnen nach \$. 10. (৫) oder nach (VIII.) gleichen gebrochenen Factoriellen; denkt man sich gleichzeitig $\nu=\infty$, um (nach XIX.) statt der Factoriellen mit unendlich grofser Basis die Potenzen setzen zu können, so findet sich augenblicklich

$$
\text { 3. } \quad 2^{a-1} \cdot \frac{1^{\frac{1}{3} a-1 \mid 1} \cdot 1^{\frac{1}{3} a-\frac{1}{3} \mid 1}}{1^{a-1 \mid 1}}=\frac{2^{\nu \mid 2}}{1^{\left.\nu\right|^{2}} \cdot \nu^{\frac{1}{2}}} \text { für } \quad \nu=\infty,
$$

so dafs auch in (3.) der Ausdruck links, von $a$ ganz unabhängig ist. Wird nun hier 1 statt $a$ gesetzt, so erhält man mittelst der Gleichungen (XI. und XXXI.)

$$
\text { 4. } \left.\quad \sqrt{ } \pi={\frac{2^{\nu \mid 2}}{1^{\nu \mid 2} \cdot \nu^{\frac{1}{3}}}}^{*}\right) \text {. }
$$

der Ausdruck unter dem Producten-Zeichen $\boldsymbol{\Pi}$, über in

$$
2 \sin \frac{\mu}{n} \pi \cdot\left(\sin \frac{\mu}{n} \pi-i \cdot \cos \frac{\mu}{n} \pi\right),
$$

während das Product der $n-1$ eingeklammerten Factoren $\sin \frac{\mu}{n} \pi-i \cdot \cos \frac{\mu}{n} \pi$ deshalb $=1$ wird, weil dies mit dem Producte je zweier, vom Anfange und vom Ende gleich weit entfernter dieser Factoren der Fall ist; denn sie sind für $\mu=r$ und $\mu=n-r$ (weil $\sin (\pi-z)=\sin z$ aber $\cos (\pi-z)=-\cos z$ ist) bezüglich

$$
\sin \frac{r}{n} \pi-i \cdot \cos \frac{r}{n} \pi \text { und } \sin \frac{r}{n} \pi+i \cdot \cos \frac{r}{n} \pi \text {. }
$$

*) Multiplicirt man diese Gleichung mit $\frac{1}{\sqrt{2}}=\left(\frac{\nu}{2 \nu+1}\right)^{\frac{1}{2}}$ für $v=\infty$, und quadrirt man sie dann, so erhält man (nach XXVII.)

$$
\frac{1}{2} \pi=\frac{2^{\left.\nu\right|^{2}} \cdot 2^{\left.\nu\right|^{2}}}{1^{v \mid 2} \cdot 3^{\left.\nu\right|^{2}}} \text { für } \nu=\infty \text {; }
$$

und dies ist der bekannte Ausdruck des Wallis für $\pi$. . 
Die Gleichung (3.) geht daher über, wenn man noch $2 a$ statt $a$ setzt, in

$$
\text { 5. } 2^{2 a-1} \cdot \frac{1^{a-1 \mid 1} \cdot 1^{a-\frac{1}{2} \mid}}{1^{2 a-1 \mid 1}}=\sqrt{ } \pi
$$

und, wenn $a$ positiv (nach XIV.), in

$$
\text { XXXV. } \quad \Gamma_{a} \cdot I_{a+\frac{\pi}{2}}^{\prime}=I_{2 a}^{\prime} \cdot 2^{-2 a+1} \cdot \sqrt{ } \pi,
$$

welche Gleichung ebenfalls eine bekannte Eigenschaft der Gamma-Functionen ist.

\$. 13.

Drittes Beispiel. Man gehe von derselben Eigenschaft der Producte äqidifferenter Factoren aus, welche aber jetzt in der allgemeinern Gleichung

1. $b^{n||^{1}}=b^{\nu \mid n} \cdot(b+1)^{\nu \mid n} \cdot(b+2)^{\nu \mid n} \ldots(b+n-1)^{\nu \mid n}$

ausgesprochen ist (nämlich, dafs sich solche Producte durch Versetzung ihrer Factoren am Werthe nicht ändern) und reducire (nach IX.) die Factoriellen mit der Differenz $n$ auf solche mit der Differenz 1, so dafs die Gleichung (1.) übergeht, wenn man gleichzeitig $n \iota$ statt $b$ schreibt, in

$$
\text { 2. } \frac{(n a)^{n v \mid 1}}{a^{v \mid 1} \cdot\left(a+\frac{1}{n}\right)^{v \mid 1} \cdot\left(a+\frac{2}{n}\right)^{v \mid 1} \cdots\left(a+\frac{n-1}{n}\right)^{v \mid 1}}=n^{n v} \text {. }
$$

Substituirt man nun hier herein wiederum statt der ganzen Factoriellen (nach \$.10. (৫) oder nach VIII.) ihre Ausdrücke in gebrochene Factoriellen, so ergiebt sich, wenn gleichzeitig $\nu=\infty$ gedacht wird, augenblicklich

3. $\quad n^{n a} \cdot \frac{1^{a-1 \mid 1} \cdot 1^{a-1+\left.\frac{1}{n}\right|^{1} \cdot 1^{a-1+\frac{2}{n} \mid} \mid 1} \ldots 1^{a-1+\frac{n-1}{n} \mid 1}}{1^{n a-1 \mid 1}}=\frac{\left(1^{v \mid 1}\right)^{n} \cdot n^{n \nu+1}}{1^{n v \mid 1} \cdot \nu^{\frac{1}{(n-1)}}}$,

so dafs der Ausdruck links, von a unabhängig wird. Ist nun a positiv, so geht dieser Ausdruck zur Linken über in

$$
\text { 4. } n^{n a} \cdot \frac{\Gamma_{a} \cdot \Gamma_{a+\frac{1}{n}} \cdot \Gamma_{a+\frac{2}{n}} \cdots \Gamma_{a+\frac{n-1}{n}}}{\Gamma_{n a}},
$$

so dafs dieser letztere auch von $a$ unabhängig ist. Weil solcher aber nach (XXXIV.) für $a=\frac{1}{n}$ den Werth $(2 \pi)^{\frac{1}{2}(n-1)} \cdot \sqrt{ } n$ annimmt, so folgt hieraus

$$
\text { XXXVI. } \quad I_{a}^{\prime} \cdot I_{a+\frac{1}{n}} \cdot \Gamma_{a+\frac{2}{n}} \ldots I_{a+\frac{n-1}{n}}^{\prime}=I_{n a}^{\prime} \cdot n^{-n a+\frac{1}{2}} \cdot(2 \pi)^{\frac{1}{2}(n-1)},
$$

in welcher Formel die (XXXV.) (für $n=2$ ) als ein besonderer Fall enthalten ist. 
Anmerkung. So sehen wir diese bekannten, aber bis jetzt ziemlich isolirt stehenden Eigenschaften der Gamma-Functionen, in einer so vernunftnothwendigen organischen Gliederung erscheinen, dafs sie nun als vollkommenes Gemeingut der ersten Elemente des Calculs angesehen werden können. - Diese letztere Behauptung rechtfertigt sich aber noch mehr, sobald man folgendes noch erwägt:

A. Die Formel

$$
\text { 1. } b^{n c \mid 1}=b^{c \mid n} \cdot(b+1)^{c \mid n} \cdot(b+2)^{c \mid n} \ldots(b+n-1)^{c \mid n}
$$

und natürlich auch der besondere Fall von ihr, wo $n=2$, nämlich

$$
\text { 2. } b^{2 c}=b^{c \mid 2} \cdot(b+1)^{\left.c\right|^{2}} \text {, }
$$

von welcher (oder von welchen) wir, indem wir $\boldsymbol{c}$ wie $n$, positiv ganz uns dachten, in den beiden vorhergehenden Paragraphen, als von der einfachsten Eigenschaft der Producte ausgegangen sind, - gilt (oder gelten) auch noch, wenn c beliebig reell ist (ganz oder gebrochen, posiliv oder negativ), wie die Anwendung der Formel (XXII.) unmittelbar auf das überzeugendste erkennen läfst.

B. Diese Formeln (1. und 2.), für gebrochene Werthe von c aufgefafst und für $b=1$, sind nichts anders als eben diese zwei zuletzt erwähnten Eigenschaften der Gamma-Functionen, wie solche in den Formeln (XXXVI. und XXXV.) zu finden sind. Die (1.) geht nämlich, sobald man $a-\frac{b}{n}$ statt $c$ setzt, sogleich (nach XV.) über in

$$
\frac{\Gamma_{n a}}{\boldsymbol{\Gamma}_{b}}=n^{n a-b} \cdot \frac{\boldsymbol{\Gamma}_{a} \cdot \boldsymbol{\Gamma}_{a+\frac{1}{n}} \cdot \boldsymbol{\Gamma}_{a+\frac{2}{n}} \ldots \boldsymbol{\Gamma}_{a+\frac{n-1}{n}}}{\boldsymbol{\Gamma}_{\frac{b}{n}} \cdot \boldsymbol{\Gamma}_{\frac{b+1}{n}} \cdot \boldsymbol{\Gamma}_{\frac{b+2}{n}} \cdots \Gamma_{\frac{b+n-1}{n}}} .
$$

C. So wie also z. B. in der Eigenschaft

$$
a^{m+n}=a^{m} \cdot a^{n}
$$

der Producte gleicher Factoren, sobald man sich $m$ und $n$ gebrochen denkt, eine Eigenschaft der Wurzeln ausgesprochen ist, - dieselbe Gleichung aber Eigenschaften der Sinus und Cosinus ausdrückt, so oft man sich $m$ und $n$ imaginär und von der Form $x \cdot \sqrt{ }-1$ und $z \cdot \sqrt{ }-1$ denkt und $a=e$ nimmt, eben so drückt jede einfache Gleichung zwischen Producten äquidifferenter Factoren, sobald man sie in Factoriellen-Zeichen schreibt, und statt der (anfänglich ganz gedachten) Exponenten nun gebrochene Zahlen sich denkt, gleichzeitig Eigenschaften der Gamma-Functionen aus. 
Dafs aber dieselbe Formel, die für ganze Exponenten gilt, auch noch für gebrochene Exponenten wahr sei, -- mufs natürlich jedesmal besonders nachgewiesen werden.

Wir wollen nun noch einige andere Betrachtungen anstellen.

S. 14.

Setzt man in die Gleichung

$$
(1+x)^{a} \cdot(1+x)^{b}=(1+x)^{a+b}
$$

statt der Potenzen die, ihnen gleichen, nach ganzen Potenzen von $x$ fortlaufenden Binomialreihen, - multiplicirt man die beiden Reihen zur Linken, und vergleicht man mit einander die Coëfficienten der $n$ ten Potenz von $x$, so erhält man eine Vergleichung der Binomial-Coëfficienten, welche, wenn man mit $1 \cdot 2 \cdot 3 \cdot 4 \ldots n$ (welches Product wir der Kürze wegen stets durch $n$ ! bezeichnen wollen) multiplicirt, zu einer Vergleichung zwischen Factoriellen mit der Differenz -1 führt, die aber in eine Vergleichung zwischen Factoriellen mit der beliebigen Differenz $\boldsymbol{r}$ übergeht, sobald man die Gleichung links und rechts mit $(-r)^{n}$ multiplicirt und, da es lauter ganze Factoriellen sind, die Formel (IX.) in Anwendung bringt.

Die so erhaltene Gleichung ist dann folgende:

$$
\text { XXXVII. } \quad(\boldsymbol{a}+\boldsymbol{b})^{n \mid r}=\sum_{\mathfrak{b}=0}^{\mathfrak{b}=n}\left(\boldsymbol{n}_{\mathfrak{b}} \cdot \boldsymbol{a}^{n-\mathfrak{b} \mid r} \cdot \boldsymbol{b}^{\mathfrak{b} \mid r}\right),
$$

wo $n_{1}, n_{1}, n_{2}, n_{3}, \ldots n_{\mathfrak{b}}, \ldots$ die Binomial-Coëfficienten der nten Potenz irgend eines Binomiums vorstellen. Dies ist der sogenannte binomische Lehrsatz für Factoriellen, der für $r=0$ in den gewöhnlichen binomischen Lehrsatz für Potenzen, übergeht. - In dieser Gleichung (XXXVII.) sind $a$ und $b$ ganz beliebig reell oder imaginär gedacht, aber $n$ positiv ganz *).

*) Für $r=1$ und $b$, so wie a positiv, geht dieser Satz (nach XV.) über in

$$
\text { (A.) } \frac{\boldsymbol{\Gamma}_{a+b+n}}{\boldsymbol{\Gamma}_{a+b}}=\sum_{\mathfrak{b}=0}^{\mathfrak{b}=n}\left(n_{\mathfrak{b}} \cdot \frac{\boldsymbol{\Gamma}_{a+n-\mathfrak{b}} \cdot \boldsymbol{\Gamma}_{b+\mathfrak{b}}}{\boldsymbol{\Gamma}_{a} \cdot \boldsymbol{\Gamma}_{b}}\right) \text {. }
$$

Zu derselben Gleichung wird man aber auch geführt, wenn man von dem Eulerschen Integral erster Classe

$$
\varphi_{a, b}=\int_{0}^{1} x^{a-1}(1-x)^{b-1} \cdot d x
$$

ausgeht, die Differential-Function zur Rechten aber mit

multiplicirt. Man erhält dann sogleich

$$
1=[x+(1-x)]^{n}=\sum_{\mathfrak{b}=0}^{\mathfrak{b}} \overline{\bar{N}}^{n}\left(n_{\mathfrak{b}} \cdot x^{n-\mathfrak{b}}(1-x)^{\mathfrak{b}}\right)
$$

$$
\text { (B.) } \quad \varphi_{a, b}=\sum_{\mathfrak{b}=0}^{\mathfrak{b}=n}\left(n_{\mathfrak{b}} \cdot \varphi_{a+n-\mathfrak{b}, b+\mathfrak{b})}\right. \text {; }
$$


So wie man sich $n$ nicht positiv ganz denkt, wird die Reihe zur Rechten (XXXVII.) (wenn nicht etwa $b$ ein entgegengesetztes Vielfaches von $r$ sein sollte, oder $n$ negativ ganz und $a$ ein directes Vielfaches von $r$ ) eine unendliche, da die Beschränkung $\mathfrak{b}=n$ nun wegfallen mufs; und es fragt sich nun, ob diese unendliche Reihe

$$
\text { (R.) ... } \Sigma\left(\boldsymbol{n}_{\mathfrak{b}} \cdot \boldsymbol{a}^{n-b \mid r} \cdot \boldsymbol{b}^{\mathrm{k} \mid r}\right) \text {, }
$$

wo $\mathfrak{b}$ nach und nach 0 und alle positiven ganzen Zahlen vorstellt, - noch immer der Factorielle $(\boldsymbol{a}+\boldsymbol{b})^{n \mid r}$ gleich sein wird, wie dies dann der Fall ist, wenn man $n$ positiv ganz nimmt (weil dann die Binomial-Coëfficienten $n_{\mathfrak{b}}$ alle der Null gleich werden, so oft $\mathfrak{b}>n$ genommen wird, so dafs die unendliche Reihe ( $\boldsymbol{R}$.) sich nun auf die endliche der (XXXVII.) reducirt). Wir werden aber finden:

1) dafs diese unendliche Reihe $\left(\boldsymbol{R}\right.$.) wirklich allemal $=(\boldsymbol{a}+\boldsymbol{b})^{n \mid r}$ ist, so oft $\boldsymbol{r}$ negativ ist und die Reihe convergent, - welches letztere allemal und nur dann der Fall ist, wenn gleichzeitig mit der Differenz $r$ auch $r-a-b$ negativ ist;

2) dafs dagegen dieselbe unendliche Reihe $(\boldsymbol{R}$.)

$$
=(a+b)^{n \mid r} \cdot \frac{\sin \frac{a}{r} \pi \cdot \sin \left(\frac{a+b}{r}+n\right) \pi}{\sin \left(\frac{a}{r}+n\right) \pi \cdot \sin \frac{a+b}{r} \pi}
$$

sein wird, so oft $\boldsymbol{r}$ positiv ist, und wenn die unendliche Reihe (R.) convergirt, welches letztere allemal und nur dann der Fall ist, wenn zugleich mit $r$ auch $r-a-b$ positiv sich findet.

Dieser letztere Ausdruck zieht sich aber auf seinen ersten Factor $(a+b)^{n \downarrow r}$ allemal zurück, so oft $n$ positiv oder negativ ganz ist, oder $b$ ein positiv oder negativ Vielfaches von $r$.

Die folgenden Paragraphen sollen dies alles aufser Zweifel stellen.

also für $n=1,2,3$, etc.

u. s. w.

$$
\begin{aligned}
& \varphi_{a, b}=\varphi_{a+1, b}+\varphi_{a, b+1} \\
& \varphi_{a, b}=\varphi_{a+2, b}+2 \cdot \varphi_{a+1, b+1}+\varphi_{a, b+2} \\
& \varphi_{a, b}=\varphi_{a+3, b}+3 \cdot \varphi_{a+2, b+1}+3 \cdot \varphi_{a+1, b+2}+\varphi_{a, b+3}
\end{aligned}
$$

Und diese Gleichung $(\boldsymbol{B}$.) geht sogleich in die vorhergehende Gleichung $(\boldsymbol{A}$.) über, sobald man die Eigenschaft der Function $\varphi$ zu Hilfe nimmt, nach welcher

ist.

$$
\text { (C.) } \quad \varphi_{p, q}=\frac{\Gamma_{p} \cdot \Gamma_{q}}{\Gamma_{p+q}}
$$

Von der Gleichung ( $\boldsymbol{B}$.) ausgehend, kann man also auch den binomischen Lehrsatz für Factoriellen erhalten, welches Verfahren jedoch nicht zu rühmen sein dürfte. 
§. 15.

Stellen wir uns zunächst die Aufgabe:

Die Summe der unendlichen Reihe

$$
\text { (R.) . . } \Sigma\left(n_{\mathfrak{b}} \cdot a^{n-\mathfrak{b} \mid r} \cdot b^{\mathfrak{b} \mid r}\right)
$$

in dem Falle zu finden, in welchem sie convergent ist *).

Man hat nach den vorher entwickelten Formeln, namentlich aber nach den wichtigsten derselben, nämlich nach der (V., VI. und XII.)

1. $\boldsymbol{a}^{n-\mathfrak{b} \mid r}=\boldsymbol{a}^{n \mid r} \cdot(\boldsymbol{a}+\boldsymbol{n} \boldsymbol{r})^{-\mathfrak{b} \mid r}=\frac{a^{n \mid r}}{(a+(n-1) \cdot r)^{\mathfrak{b} \mid-r}}=\frac{a^{n \mid r}}{(-1)^{b} \cdot(-a-(n-1) \cdot r)^{\mathfrak{b} \mid r}}$,

2. $\quad n_{\mathfrak{b}}=\frac{n^{\mathfrak{b} \mid-1}}{\mathfrak{b} !}=(-1)^{\mathfrak{b}} \cdot \frac{(-n)^{\mathfrak{b} \mid 1}}{\mathfrak{b} !}$, wo $\mathfrak{b} !=1^{\mathfrak{b} \mid 1}$;

also wird die Reihe (R.) so:

$$
\text { 3. } \boldsymbol{R}=\boldsymbol{a}^{n \mid r} \cdot \Sigma\left(\frac{(-n)^{\mathfrak{b} \mid 1}}{\mathfrak{b} !} \cdot \frac{b^{\mathfrak{b} \mid r}}{(-a-(n-1) \cdot r)^{\operatorname{blr}}}\right),
$$

oder, wenn man rechts Zähler und Nenner durch $\boldsymbol{r}^{\mathfrak{b}}$ dividirt, dabei die Formel (IX.) anwendet (welches, da $\mathfrak{b}$ positiv ganz oder Null ist, geschehen kann) und noch

4. $-n=\alpha ; \quad \frac{b}{r}=\beta$, so wie $-\frac{a}{r}-(n-1)=\gamma$

setzt,

$$
\text { 5. } \quad \boldsymbol{R}=\boldsymbol{a}^{n \mid r} \cdot \Sigma\left(\frac{\alpha^{\mathfrak{b} 1} \cdot \beta^{\mathfrak{b} \mid 1}}{\mathfrak{b} ! \gamma^{\mathfrak{b} \mid 1}}\right) .
$$

Nun ist aber diese letztere unendliche Reihe genau die von Gau/s in der oben bereits angeführten Abhandlung (1812) behandelte, und wir wissen aus dieser Behandlung, dafs sie nur dann, aber dann auch allemal convergent ist, so oft $\gamma-\alpha-\beta$ d. h. $1-\frac{a+b}{r}$ oder $\frac{r-a-b}{r}$ positiv ist, also wenn $r$ mit $r-a-b$ entweder zugleich negativ, oder zugleich positiv ist.

\section{§. 16.}

Suchen wir nun die Summe

6. $\boldsymbol{S}_{\gamma}$ der unendlichen Reihe $\Sigma\left(\frac{\alpha^{\mathfrak{b} \mid 1} \cdot \beta^{\mathfrak{b} \mid 1}}{\mathfrak{b} ! \gamma^{\mathfrak{b} 1}}\right)=\boldsymbol{F}_{\alpha, \beta, \gamma}$.

*) Warum man hier (wie allemal, wo von Werthen die Rede ist, welche für specielle Werthe eines Buchstaben dadurch hervorgegangen sind, dafs man etwa $\frac{1}{\infty}=0$ oder dergl. gesetzt hat) die Bedingung der Convergenz stellen müsse, die au/serdem. sehr überflïssig ist, geht aus pag. 20 N. 23. des „Geistes der Diff. und Integral-Rechnung etc. Erlangen 1846." hervor. 
Da diese Summe dem allerersten Gliede 1 der Reihe sich desto mehr nähert, je gröfser $\gamma$ gegen $\alpha$ und $\beta$ ist, so kommt alles darauf an, $\boldsymbol{S}_{\gamma}$ in $\boldsymbol{S}_{\gamma+1}$ auszudrücken, um mittelst dieser Relation auch $\boldsymbol{S}_{\gamma}$ in $\boldsymbol{S}_{\gamma+\nu}$ ausdrücken zu können, während $S_{\gamma+\nu}=1$ wird für $\nu=\infty$. - Man thut aber gut, statt der unendlichen Reihe stets eine Reihe von $\mu$ Gliedern zu nehmen, für diesen Fall die Rechnungen zu machen, und zuletzt erst $\mu=\infty$ sich zu denken, um mit der Convergenz der Reihen nicht Schwierigkeiten zu haben, während die endlichen Reihen, wenn man sich der so höchst einfachen "Theorie der combinat. Aggregate" bedient, wie solche im 2ten Theile des „Versuchs eines etc. etc. Systems der Mathematik, 2te Auflage," entwickelt steht, dann genau dieselben bequemen Rechnungen geben, wie wenn die Reihen unendliche sind. - Wir wollen uns jedoch hier dieses Rechnungsvortheils begeben, bekommen aber dadurch an einer Stelle die Differenz zweier unendlichen Reihen, die beide unter den gemachten Voraussetzungen nicht nothwendig convergent sind. Der geneigte Leser wolle also, im Falle sich ihm Bedenken ergeben sollten, die Rechnungen mit endlichen Reihen wiederholen.

Man findet, indem die Glieder der Reihe $\boldsymbol{F}_{\alpha, \beta, \gamma}$ mit $\gamma-\alpha-1=$ $(\boldsymbol{\gamma}+\mathfrak{b}-1)-(\alpha+\mathfrak{b})$ multiplicirt werden, zunächst

$$
\text { 7. } \frac{\gamma-\alpha-1}{\gamma-1} \cdot \boldsymbol{F}_{\alpha, \beta, \gamma}=\boldsymbol{F}_{\alpha, \beta-1, \gamma-1},
$$

und, wenn man hier $\gamma-1$ statt $\gamma$ und $\beta-1$ statt $\alpha$, so wie $\alpha$ statt $\beta$ schreibt, und wenn man bedenkt, dafs die beiden erstern Elemente in $\boldsymbol{F}$ stets mit einander vertauscht werden können,

$$
\text { 8. } \frac{\gamma-\beta-1}{\gamma-2} \cdot \boldsymbol{F}_{\alpha, \beta-1, \gamma-1}=\boldsymbol{F}_{\alpha-1, \beta-1, \gamma-2}
$$

folglich, wenn man diese beiden Gleichungen mit einander multiplicirt,

$$
\text { 9. } \quad \frac{(\gamma-\alpha-1)(\gamma-\beta-1)}{(\gamma-1)(\gamma-2)} \cdot \boldsymbol{H}_{\alpha, \beta, \gamma}=\boldsymbol{F}_{\alpha-1, \beta-1, \gamma-2} \text {. }
$$

Ferner findet sich, wenn man die (6.) mit $\frac{\beta-1}{\gamma-1}$ multiplicirt, und das Product von der (7.) subtrahirt,

10. $\frac{\gamma-\alpha-\beta}{\gamma-1} \cdot \boldsymbol{F}_{\alpha, \beta, \gamma}=\boldsymbol{F}_{\alpha-1, \beta-1, \gamma-1}$.

Wird nun in (9.) $\gamma+1$, statt $\gamma$ gesetzt, und das Resultat mit der (10.) verglichen, so findet sich noch

$$
\text { 11. } \boldsymbol{F}_{\alpha, \beta, \gamma}=\frac{(\gamma-\alpha) \cdot(\gamma-\beta)}{\gamma \cdot(\gamma-\alpha-\beta)} \cdot \boldsymbol{F}_{\alpha, \beta, \gamma+1},
$$

welches die wichtige von uns gesuchte Relation ist.

Setzt man nun in sie statt $\gamma$ nach und nach $\gamma+1, \gamma+2, \ldots \gamma+\nu-1$, und multiplicirt man die entstehenden Gleichungen alle mit einander und mit 
der (11.), nimmt man zuletzt $\nu=\infty$, damit $\boldsymbol{F}_{\alpha, \beta, \gamma+\nu}=1$ wird, so hat man

12. $F_{\alpha, \beta, \gamma}$ oder $\boldsymbol{S}_{\gamma}=\frac{(\gamma-\alpha)^{\nu \mid 1} \cdot(\gamma-\beta)^{\nu \mid 1}}{\gamma^{\nu \mid 1} \cdot(\gamma-\alpha-\beta)^{\nu \mid 1}}$ für $\nu=\infty$.

Diese ganzen Factoriellen kann man nun aber nach $\$$. 10. $(\odot)$ oder nach (VIII.) sogleich wieder in allgemeinere Factoriellen umformen, und man findet dann ohne Weiteres

$$
\text { XXXVIII. } \quad \Sigma\left(\frac{\alpha^{\mathfrak{b} \mid 1} \cdot \beta^{\mathfrak{b} \mid 1}}{\mathfrak{b} ! \gamma^{\mathfrak{b}}}\right)=\frac{(\gamma-\alpha)^{\alpha \mid 1}}{(\gamma-\alpha-\beta)^{\alpha \mid 1}}=\frac{(\gamma-\beta)^{\beta \mid 1}}{(\gamma-\alpha-\beta)^{\beta \mid 1}} * \text {. }
$$

Dies Resultat in die (5.) substituirt, und wenn statt $\alpha, \beta, \gamma$ wieder ihre Werthe aus (4.) gesetzt werden, giebt dann unsere gesuchte Reihe (R.), nämlich XXXIX. $\Sigma\left(n_{\mathfrak{b}} \cdot a^{n-b \mid r} \cdot b^{\mathfrak{k} \mid r}\right)=a^{n \mid r} \cdot \frac{\left(1-\frac{a}{r}\right)^{-n \mid 1}}{\left(1-\frac{a+b}{r}\right)^{-n \mid 1}}=\frac{\left(-\frac{a+b}{r}\right)^{n \mid-1}}{\left(-\frac{a}{r}\right)^{n \mid-1}}=\frac{\left(1-n-\frac{a+b}{r}\right)^{n \mid 1}}{\left(1-n-\frac{a}{r}\right)^{n \mid 1}}$, welches in allen Fällen, wo $r$ mit $r-a-b$ einerlei Vorzeichen hat, die Summe der vorgelegten (convergenten) Factoriellen- (Binomial-) Reihe ist.

\section{\$. 17.}

Ist nun

1) r negativ, also - $r$ positiv, so kann man in dem zuletzt gefundenen Ausdrucke, Zähler und Nenner mit $(-r)^{n}$ multipliciren und die Formel (IX.) anwenden, und dann geht sogleich

$$
\text { XL. } \quad \Sigma\left(n_{\mathfrak{b}} \cdot a^{n-b \mid r} \cdot b^{\mathfrak{b} \mid r}\right)=(a+b)^{n \mid r}
$$

hervor.

Ist dagegen

2) $r$ positiv, so gilt dieses Verfahren nicht mehr (weil die (IX.) nur gilt, so lange das dortige $h$ positiv ist) und man kann dann die (XXXIX.) so schreiben:

*) $\mathrm{Da}$ (nach VII.) $(\gamma-\alpha)^{\alpha \mid 1}=\frac{1^{\gamma-1 \mid 1}}{1^{\gamma-\alpha-1 \mid 1}}$ und $(\gamma-\alpha-\beta)^{\alpha \mid 1}=\frac{1^{\gamma-\beta-1 \mid 1}}{1^{\gamma-\alpha-\beta-1 \mid 1}}$ ist, so kann man die so eben gefundene Summe auch so schreiben:

$$
\frac{1^{\gamma-1 \mid 1} \cdot 1^{\gamma-\alpha-\beta-1 \mid 1}}{1^{\gamma-\alpha-1 \mid 1} \cdot 1^{\gamma-\beta-1 \mid 1}}
$$

und dies stimmt genau mit dem von Gau/s in der angeführten Abhandlung für dieselbe Summe gefundenen Ausdruck

überein.

$$
\frac{\Pi_{\gamma-1} \cdot \Pi_{\gamma-\alpha-\beta-1}}{\Pi_{\gamma-\alpha-1} \cdot \Pi_{\gamma-\beta-1}}
$$

Sind $\gamma, \gamma-\alpha$ und $\gamma-\beta$ auch noch positiv, so geht dieselbe Summe nach S. 4. (XIV. oder XV.) über in

$$
\frac{\boldsymbol{T}_{\gamma} \cdot \boldsymbol{I}_{\gamma-\alpha-\beta}}{\boldsymbol{\Gamma}_{\gamma-\alpha} \cdot \boldsymbol{\Gamma}_{\gamma-\beta}}
$$

Crelle's Journal f. d. M. Bd. XXXVI. Heft 4. 


$$
\begin{aligned}
\Sigma\left(n_{\mathfrak{b}} \cdot a^{n-\mathfrak{b} \mid r} \cdot b^{\mathfrak{b} \mid r}\right) & =(a+b)^{n \mid r} \cdot \frac{a^{n \mid r}}{(a+b)^{n \mid r}} \cdot \frac{\left(1-\frac{a}{r}\right)^{-n \mid 1}}{\left(1-\frac{a+b}{r}\right)^{-n \mid 1}} \\
& =(a+b)^{n \mid r} \cdot \frac{\left(\frac{a}{r}\right)^{n \mid 1} \cdot\left(1-\frac{a}{r}\right)^{-n \mid 1}}{\left(\frac{a+b}{r}\right)^{n \mid 1} \cdot\left(1-\frac{a+b}{r}\right)^{-n \mid 1},}
\end{aligned}
$$

d. h. (nach IX.)

oder (nach XXIV.)

$$
\text { XLI. } \quad \sum\left(n_{\mathfrak{b}} \cdot a^{n-b \mid r} \cdot b^{\mathfrak{b} \mid r}\right)=(a+b)^{n \mid r} \cdot \frac{\sin \frac{a}{r} \pi \cdot \sin \left(\frac{a+b}{r}+n\right) \pi}{\sin \left(\frac{a}{r}+n\right) \pi \cdot \sin \frac{a+b}{r} \pi} \text {. }
$$

Alles dieses ist aber im $\$$. 14 . behauptet worden und nun strenge erwiesen.

$$
\text { \$. } 18 .
$$

Namentlich ist also (für $r=-1$ und $r=+1$ )

$$
\text { XLII. } \quad \Sigma\left(n_{\mathfrak{b}} \cdot a^{n-\mathfrak{b} \mid 1} \cdot b^{\mathfrak{b} 11}\right)=(a+b)^{n \mid-1}
$$

für jeden beliebigen reellen Werth von $n$, wenn nur, der Convergenz wegen, $a+b+1$ positiv ist; und ferner ist noch

$$
\text { XLIII. } \quad \Sigma\left(n_{\mathfrak{b}} \cdot a^{n-\mathfrak{b l 1}} \cdot b^{\mathfrak{b} \mid 1}\right)=(a+b)^{n \mid 1} \cdot \frac{\sin a \pi \cdot \sin (a+b+n) \pi}{\sin (a+n) \pi \cdot \sin (a+b) \pi}
$$

für jeden beliebigen reellen Werth von $n$, wenn nur, der Convergenz wegen, 1-a-b positiv ist. - Dieser Ausdruck zur Rechten ist aber wieder von $(a+b)^{n \mid 1}$ nicht mehr verschieden, so oft $\boldsymbol{b}$ oder $n$ positiv oder negativ ganz ist.

Diese Resultate (XLI. und XLIII.) irgend wo bereits gesehen zu haben, erinnern wir uns nicht. - Kramp hält die Gleichung (XL.) noch für allgemein wahr, eben sowohl für negative wie für positive Werthe von $r$, welches jedoch, wie wir so eben gesehen haben, ein Irrthum ist. - Gau/s endlich hat in seiner, oben angeführten trefflichen Abhandlung nur die einfachste Factorielle $1^{c 11}$ betrachtet, und diese nur aus dem Gesichtspuncte einer transcendenten Function $\Pi_{c}$ von $c$. Von diesem Standpuncte aus konnte der binomische Lehrsatz für Factoriellen gar nicht in Untersuchung kommen, obgleich das Material dazu auch in dieser Gau/sischen Abhandlung nicht vergebens gesucht wird, sobald man nur dazu den einfacheren und freieren Standpunct erwählt hat.

\section{\$. 19.}

$\mathrm{Zu}$ den einfachsten Mitteln bestimmte Integrale auszuwerthen, gehört noch immer die Umformung der Differential-Function in Reihen, die nachfolgende Integration dieser letzteren zwischen den vorgegebenen Grenzen, und die endliche Summation der gewonnenen Resultate, um das Endresultat wie- 
derum in endlicher Form zu erhalten. - Aus diesem Gesichtspuncte haben Kramp (1799) und Gau/s (1812) in den oben angeführten Abhandlungen eine Anzahl bestimmter Integrale, der erstere in die Binomialreihe für Factoriellen, der letztere in die von ihm behandelte Reihe $\Sigma\left(\frac{\alpha^{\mathfrak{b} 11} \cdot \beta^{\mathfrak{b} \mid 1}}{\mathfrak{b} ! \gamma^{\mathfrak{b} \mid 1}}\right)$ (auf welche sich die erstgedachte Binomialreihe zurückführen läfst) ausgedrückt, während bei beiden die Summation dieser Reihen zu gebrochenen Factoriellen führt, nur dafs sie Gau/s weder so nennt, noch so bezeichnet, als Kramp es gethan. - Wir berühren dies hier nur als etwas bekanntes, heben aber besonders hervor, dafs auf diesem Wege eine gröfsere Anzahl bestimmter Integrale in gebrochene Factoriellen also auch in Gamma-Functionen ohne Weiteres sich ausdrücken läfst, und da/s deshalb die Theorie der Facloriellen auch die wichligsten Eigenschafien jener neuen Transcendenten in sich schliefst, also $\approx$. B. auch die Eigenschaflen des von uns oben durch $\varphi_{a, b}$ bezeichneten Euler'schen Inlegrals 1ler Klasse $\int_{0}^{1} x^{a-1}(1-x)^{b-1} \cdot d x$.

Auch die numerische Berechnung der Gamma-Functionen entwickelt sich am einfachsten und naturgemälsesten, wenn man sie aus dem Gesichtspuncte der Factoriellen betrachtet.

Wir wollen jedoch dies alles, wie so vieles, was sich noch daran anreiht, hier nicht weiter verfolgen, sondern eine ausführlichere Behandlung einer eigenen Schrift aufbewahren. Unser gegenwärtiger Zweck ist erreicht, wenn diese kleine Abhandlung beiträgt, das Vorurtheil zu entfernen, nach welchem die Behandlung der allgemeineren Factoriellen, als aggregirender Bestandtheil der Elemente der Analysis deshalb erlassen werden könne, weil sich alle Factoriellen auf die einfachste derselben $1^{c \mid 1}$ oder $\Pi_{c}$ zurückführen lassen, d. h. auf eine Function eines einzigen Veränderlichen. - Mit gröfserem Rechte könnte man aber dann aus den Elementen auch den Gebrauch der Potenz $\boldsymbol{x}^{z}$ entfernen, weil sich alle Potenzen auf die natürliche $e^{y}$ zurückführen lassen, d. h. auf die Function eines einzigen Veränderlichen, in so fern $e$ als Basis der natürlichen Logarithmen ein bestimmter Ziffernwerth ist.

Je mehr die Wissenschaften mit der Zeit aus einander gehen und sich vereinzeln, desto mehr thut es Noth, dafs von Zeit zu Zeit der Versuch gemacht werde, ob sich nicht das scheinbar Vereinzelte und Getrennte auch wieder einem organisch gegliederten Ganzen anreihen lasse. Erst nachdem dieses geglückt ist, kann man sich des sicheren und bleibenden Besitzes desselben erfreuen. ,

Berlin, im März 1848. 